\title{
SELBSTHILFE
}

\section{Empowerment organisieren}

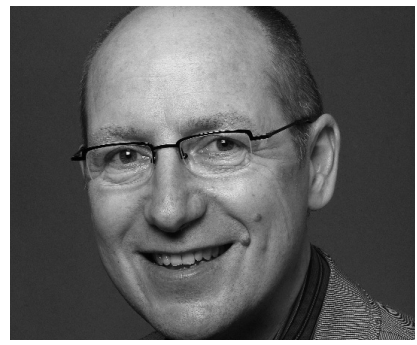

VON ANDREAS GREIWE Andreas Greiwe ist Leiter des Projekts »In-Gang-Setzer « und Fachberater Selbsthilfe und SelbsthilfeKontaktstellen beim

Paritätischen Wohlfahrtsverband in Nordrhein-Westfalen.

E-Mail greiwe@paritaet-nrw.org

\author{
Das Projekt "In-Gang-Setzer ${ }^{\circledR}$ " unterstützt Menschen, \\ die eine Selbsthilfegruppe gründen wollen, denn gerade \\ deren Anfangszeit ist von Unsicherheit und Verletzbar- \\ keit geprägt. An Selbsthilfe interessierten Personen feh- \\ len oft die kommunikativen und organisatorischen \\ Fähigkeiten, um diese sensible Startphase konstruktiv \\ zu gestalten. Hier setzt das aus Dänemark stammende \\ Konzept an.
}

Durchschnittlich nur fünf bis sieben Prozent der von einem gesundheitlichen oder sozialen Problem betroffenen Menschen engagieren sich aktuell in Selbsthilfegruppen. Gemessen an der geäußerten Bereitschaft, sich bei Bedarf einer Gruppe anzuschließen, ist das Potential damit nicht ausgeschöpft.

Die Teilnahme an einer Selbsthilfegruppe bietet mit der gegenseitigen emotionalen Unterstützung eine wirksame Hilfe gegen Vereinzelung und Ausgrenzung. Insbesondere bei chronischen, psychosomatischen und Suchterkrankungen zeigt sich, dass Selbsthilfe zu mehr Wohlbefinden, einem positiven Krankheitsverlauf und längerer Lebensdauer beitragen kann. Die heilsame Kraft der Gruppe, der Gemeinschaft, kann so erlebbar werden. Dafür ist das vertraute Gespräch von herausragender Bedeutung.

Was ist aber, wenn diese Grundlage nicht gegeben ist, wenn mangelnde kommunikative Fähigkeiten und Erfahrungen den Zugang zur Selbsthilfe erschweren oder verhindern?

Der Ansatz des Projekts »In-GangSetzer®« wurde speziell zur Unterstützung neuer Selbsthilfegruppen entwickelt und hat sich als hilfreiche Methode der Selbsthilfeaktivierung erwiesen. In letzter Zeit nimmt die Bereitschaft zu, unvoreingenommen und offen zu erörtern, wie stärker als bisher dazu beigetragen werden kann, die Wege zur Selbsthilfe zu ebnen und Zugangsbarrieren abzubauen. Es geht darum, die Bereitschaft vieler Menschen, sich bei Bedarf einer Gruppe anzuschließen, konstruktiv aufzunehmen. Diese Haltung ist prägend für das Projekt »InGang-Setzer «.

\section{Das Konzept der "ln-Gang-Setzer»}

Die Anfangsphase einer Gruppe ist vielfach eine Zeit der Unsicherheit und Verletzbarkeit. Gerade den an Selbsthilfe interessierten Personen fehlen oft die Voraussetzungen, die Startphase einer Gruppe konstruktiv zu gestalten. Für viele an Selbsthilfe Interessierte würde deshalb eine Begleitung eine große Ermutigung darstellen.

Selbsthilfe-Kontaktstellen registrieren eine "große Anzahl von Nachfragen zu Themen der Lebensbewältigung und psychischen Erkrankungen und Belastungen (...) Diese Tendenz ist nicht neu, erfordert aber für die Selbsthilfe und die unterstützende Selbsthilfearbeit in den nächsten Jahren gegebenenfalls erweiterte Arbeitsansätze und langfristig wirksame, stützende Methoden «(Selbsthilfe-Kontaktstelle Bielefeld).

Es stellt sich die Frage, inwieweit es möglich ist, eine Unterstützung anzubie- 
ten, die dem Gedanken der Selbsthilfe entspricht und nachhaltig zur Selbsthilfe aktiviert. Aus Dänemark kam hierzu der Impuls der »In-Gang-Setzer «. Diese sind "Stützen für neue Selbsthilfegruppen «, sie »halfen bei praktischen Fragen beim Start einer Gruppe und brachten die Teilnehmer dazu, miteinander zu reden und sich vertraut zu fühlen. Danach zog sich der >In-Gang-Setzer〈 zurück. « (Gamst 1991)

Seit 2005 haben wir im Paritätischen Wohlfahrtsverband in Nordrhein-Westfalen die Idee aufgenommen und das Konzept auf die Situation in Deutschland übertragen. Das bundesweite Projekt »In-Gang-Setzer « begann im Dezember 2006 und wurde inzwischen bis Ende 2012 verlängert. Es wird durchgeführt vom Paritätischen Wohlfahrtsverband, Landesverband Nordrhein-Westfalen e. V. Die Projektfinanzierung haben die Betriebs-Krankenkassen übernommen, der BKK-Bundesverband und der BKK Landesverband NORDWEST.

Das Projekt »In-Gang-Setzer « umschreibt ein methodisches Konzept der
Unterstützung von Selbsthilfegruppen und ist markenrechtlich geschützt. In drei Projektphasen wurden sukzessive 22 Kontaktstellen in sechs Bundesländern einbezogen, circa 200 Personen wurden inzwischen als »In-Gang-Set- und eröffnet vielfältige Entwicklungschancen. Selbsthilfegruppen haben sich als eine neue Sozialform etabliert, die Selbstentfaltung und Selbstbestimmung ebenso ermöglicht wie Gemeinschaft, Solidarität, Rat, Trost, Unter-

\section{"Aufgabe ist die Prozessbegleitung, nicht die Ergebnisberatung"}

zer" geschult. Aktuell wird das Hauptaugenmerk auf die Gründungsphase von Gruppen gelegt. Die Erweiterung des Ansatzes als Hilfe auch für bereits aktive Gruppen wird zukünftig verstärkt einbezogen.

Selbsthilfe ist "gelungene Selbstsorge « (Heiner Keupp), sie ist keine Therapie, hat oft aber therapeutische Wirkungen. »Die soziale Nähe von Menschen in Gruppen ist von besonderer Intensität stützung und Hilfe (...) Mobilisiert wird ein großes Potenzial an sozialen Problemlösungsperspektiven. «(Thiel 1999)

Daran knüpft das Konzept der InGang-Setzung an: das Vertrauen auf das besondere Potenzial von Menschen, die sich in Selbsthilfegruppen engagieren wollen. Es geht um Empowerment, »das Zutrauen in die Fähigkeiten, die nur entfaltet werden können, wenn wir sie bei anderen Menschen voraussetzen.«

\section{Wie das Konzept »In-Gang-Setzer" funktioniert}

Die Stärken des Konzepts »In-Gang-Setzer« ruhen auf drei Säulen:

- Die Methode der »In-Gang-Setzung « hat sich bewährt bei der Gründung von neuen Selbsthilfegruppen. Diese werden durch »In-Gang-Setzer in enger Anbindung an die Kontaktstelle unterstützt, die Gruppen erhalten in der oft schwierigen Startphase eine hilfreiche Aktivierung und Orientierung.

- Der Projektverlauf zeigt, dass darüber hinaus die Methode neue Möglichkeiten eröffnet, wenn kein Initiator vorhanden ist, aber der Bedarf nach einer neuen Selbsthilfegruppe aufgrund der Nachfrage bei der Kontaktstelle offensichtlich ist.

- Auch für bereits bestehende Gruppen, die zeitlich begrenzt eine Unterstützung wünschen (Generationswechsel, Mitgliederfluktuation etc.), kann die Selbsthilfe-Kontaktstelle mit dem Konzept eine neue Form der nachhaltigen Hilfestellung anbieten.

Gegenwärtig ist der Ansatz »In-Gang-Setzung« konzeptionell und organisatorisch eng mit den lokalen Selbsthilfe-Kontaktstellen verbunden. Eine Anpassung an andere Strukturen ist Teil der Projektüberlegungen. Aufgrund von Anfragen interessierter Selbsthilfeorganisationen wird geprüft, ob-und wenn ja, unter welchen Rahmenbedingungen - diese Methode auch dort hilfreich sein kann. Gleiches gilt für die Frage, inwieweit der Ansatz der »In-Gang-Setzung" generell bei der Aktivierung von Selbsthilfe (z. B. bei Migrantinnen und Migranten) und beim Bürgerengagement allgemein hilfreich sein kann.
Derzeit erproben 22 Selbsthilfe-Kontaktstellen aus sechs Bundesländern den Ansatz:

- Nordrhein-Westfalen: Bielefeld, Detmold, Emsdetten, Gütersloh, Krefeld, Lünen, Minden, Moers, Paderborn, Recklinghausen

- Niedersachsen: Hannover, Meppen, Osnabrück, Wolfsburg

- Baden-Württemberg: Freiburg, Heilbronn, Karlsruhe

- Mecklenburg-Vorpommern: Schwerin, Stralsund

- Sachsen: Chemnitz, Dresden

- Bayern: Nürnberg

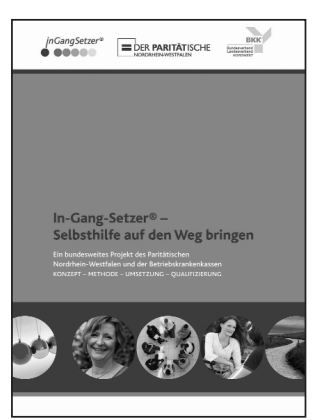

Mit dem Handbuch »In-Gang-Setzer « (92 Seiten) wird eine Bilanz der bisherigen Projekterfahrungen gezogen und aufgezeigt, wie »In-Gang-Setzer « zur nachhaltigen Aktivierung von Selbsthilfe beitragen können. Es enthält viele Arbeitshilfen, die auch außerhalb des Ansatzes für Selbsthilfegruppen hilfreich sein können. Einzelne Druckexemplare der Veröffentlichung können kostenfrei bestellt werden (E-Mail gesundheitsmedien@bkk-bv.de). Die Broschüre steht auch als PDF-Dokument zum kostenfreien Herunterladen zur Verfügung (Internet http://www.in-gang-setzer.de).

Andreas Greiwe 
(Bobzien/Stark) Dies Vertrauen in die Fähigkeiten der Menschen darf jedoch nicht den Blick verstellen auf die Hemmnisse, die viele Menschen davon abhalten, den Weg zur Selbsthilfegruppe zu finden.

Das Projekt hat das Ziel, eine Antwort auf die Frage zu geben: Trägt die Ausgangshypothese, nach der ehemalige Mitglieder von Selbsthilfegruppen oder mit Selbsthilfe vertraute und geschulte Ehrenamtliche eine besondere Akzeptanz finden bei den Menschen, die sich in einer Selbsthilfegruppe engagieren möchten?

\section{Die Aufgaben der "In-Gang-Setzer"}

Menschen, die nach dem »In-Gang-Setzer«-Konzept qualifiziert sind, können dazu beitragen, dass

- schwierige Phasen im Engagement insbesondere in der Gründungsphase - besser bewältigt werden und

- sich dadurch die Chancen für ein stabiles Engagement erhöhen.

»In-Gang-Setzer « sind ehrenamtliche Mitarbeiterinnen und Mitarbeiter der Selbsthilfe-Kontaktstellen:

- Sie stehen - gemeinsam mit der Kontaktstelle - zeitlich begrenzt den Gruppen in der Startphase zur Seite.

- Sie unterstützen die Teilnehmerinnen und Teilnehmer dabei, miteinander $\mathrm{zu}$ reden und sich vertraut zu machen sowie achten auf die Rahmenbedingungen; an der inhaltlichen Arbeit der Gruppe beteiligen sie sich nicht.

Die besondere Herausforderung für »InGang-Setzer « besteht darin, sich hilfreich in die Gruppenprozesse einzubringen, ohne sich einzumischen und Potenziale zu verschütten. Von Anfang an war daher ein Wesenszug des Konzeptes: Die »In-Gang-Setzer « dürfen sich in keiner Weise in den themenbezogenen Austausch der Gruppe einbringen! Die inhaltliche Abstinenz ist Schutz für die »InGang-Setzer «wie für die Gruppen. Es dürfen keine neuen Abhängigkeiten geschaffen werden, vielmehr muss der Boden bereitet werden für eine Gruppe, die nach einer zeitlich begrenzten Begleitung alleine ihren eigenen Weg findet.

In diesen Vorgaben unterscheidet sich der Ansatz der »In-Gang-Setzung « fundamental von Paten-Modellen, wo in Selbsthilfe erfahrene Betroffene andere, von dem gleichen Anliegen Betroffene bei der Gruppenarbeit unterstützen. Solche Patenschaften haben sich mancherorts durchaus bewährt, bergen aber die Gefahr, dass Abhängigkeiten entstehen und damit nachhaltiges Selbsthilfeengagement verschüttet wird.

Aufgabe der »In-Gang-Setzer « ist eine Prozessbegleitung, nicht eine Ergebnisberatung. »Die >In-Gang-Setzer $<$ helfen, den Gruppenprozess in Gang zu setzen, indem sie betonen, was allen gemeinsam ist. "(Gamst 1991) Es geht um Unterstützung auf den Gebieten, wo Kenntnisse und Erfahrungen fehlen (»Wie funktioniert Gruppe? «), nicht um Anleitung dort, wo Kompetenzen und Ressourcen vorhanden sind (»Experten in eigener Sache «).

Zurzeit erproben 22 Selbsthilfe-Kontaktstellen aus sechs Bundesländern den Ansatz. Es werden Vorgaben für die Auswahl der »In-Gang-Setzer «, deren Schulung und Begleitung und den organisatorischen Rahmen ihres Einsatzes entwickelt und erprobt. »In-Gang-Setzer « arbeiten ehrenamtlich; sie erhalten keine pauschale Aufwandsentschädigung, lediglich Fahrtkosten werden erstattet.

\section{Perspektiven}

Es liegen gesicherte Erkenntnisse zu der ersten Projektphase vor (bis Anfang 2009); die Zeit danach wird derzeit erfasst.

Der Anteil der Gruppen, die nach Beendigung der »In-Gang-Setzung " weiterbestehen, ist hoch (d. h. deutlich höher als bei den üblichen Gruppengründungen durch Kontaktstellen, insbesondere wenn bedacht wird, dass der Schwerpunkt auf den psychosozialen Problemen liegt). Insgesamt sind bisher rund 300 Gruppengründungen begleitet worden. Je nach Bereich schwanken die Angaben zu den erfolgreichen »InGang-Setzungen « zwischen 60 und 85 Prozent. Erfolgreich heißt: Die Gruppe existiert auch nach der »In-Gang-Setzung « noch mindestens ein halbes Jahr und hat sich in die Kartei der Kontaktstelle aufnehmen lassen. Der Großteil der Gruppen ist deutlich länger als ein halbes Jahr aktiv, viele haben sich nach Angaben der Kontaktstellen inzwischen etabliert.
Die Anliegen und Themen der Gruppen sind schwerpunktmäßig im Bereich psychische Erkrankungen und Probleme zu finden: Ängste, Panik, Depressionen, Kommunikation, emotionale Probleme, Trennung, Suizid. Dies entspricht den Ausgangsthesen und belegt die Tragfähigkeit des Ansatzes. Überraschend groß war die Zahl der AngehörigenGruppen, herausragend die »Pflegenden Angehörigen «. Möglicherweise besteht hier ein besonderer Unterstützungsbedarf, gerade mit Blick auf die demografischen Entwicklungen. Nicht selten kommen neue Selbsthilfegruppen nicht zustande, weil es an einer Person fehlt, die bereit ist, in der Anfangsphase die Initiative zu ergreifen und Verantwortung zu übernehmen. Der Ansatz der »InGang-Setzung « hat auch hier neue Perspektiven eröffnet.

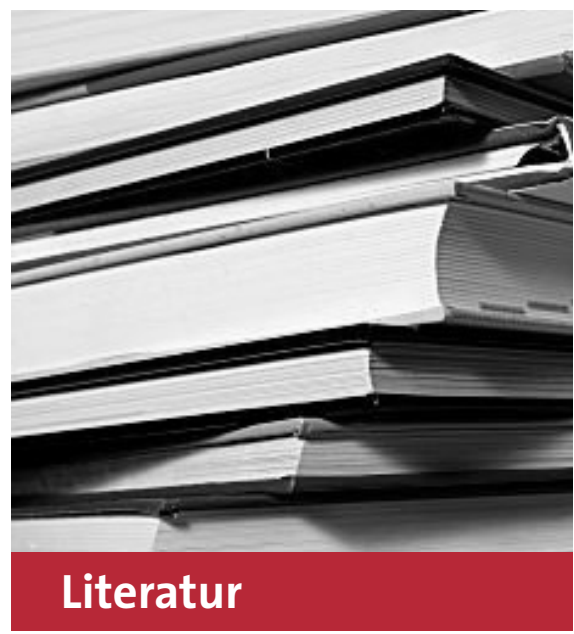

Gamst, Ann: Selbsthilfe-Arbeit bei SR-Bistand in Kopenhagen. In: DAG SHG e. V. (Hg.): Drehscheibe Kontaktstelle. Dokumentation Fachtagung 22.-24. Mai 1991 in Kiel, Gießen 1991, S. 15-22.

Greiwe, Andreas: "In-Gang-Setzer « - Stütze für neue Selbsthilfegruppen: ein bundesweites Projekt des Paritätischen NRW und der Betriebskrankenkassen (BKK). In: Kohler, Susanne/Kofahl, Christopher/Trojan, Alf (Hg.): Zugänge zur Selbsthilfe. Ergebnisse und Praxisbeispiele aus dem Projekt "Aktivierung von Selbsthilfepotentialen «, Bremerhaven 2009, S. 141-155. 